\title{
Research on MVB conformance test system
}

\author{
Jinxue Cui*, and Bin Han \\ Institute of Rail Transit, Tongji University, Shanghai, China
}

\begin{abstract}
The design and implementation of the MVB conformance test system is of great significance in both professional theory and practical application. Conformance test for MVB, mainly to determine whether the MVB equipment IUT is consistent with the MVB protocol standard requirements in the TCN standard. The conformance test of MVB equipment IUT covers most of the contents of the RTP real-time protocol such as the physical layer, link layer, network layer, transport layer and application layer. This subject will analyse and study the consistency test of the MVB physical layer.
\end{abstract}

Keywords: MVB, Conformance test, TCN.

\section{Introduction}

With the widespread application of TCN networks in the field of domestic rail transit, many equipment manufacturers design and produce communication equipment in accordance with relevant standards. In order to achieve interoperability of equipment produced by different manufacturers, communication network equipment must be completely consistent with the standard requirements [1]. Conformance testing can ensure the consistency of a tested device with the requirements of the standard, and provides the necessary guarantee for interoperability between devices. Conformance testing can also verify which aspects of a device does not meet the requirements of the corresponding standard, which is helpful for the improvement of device design. In 2020, my country issued the GB/T28029 series of standards for TCN, in which GB/T28029.10-2020 [2] describes the MVB conformance test.

\section{MVB (Multifunction Vehicle Bus)}

TCN (Train Communication Network) train communication network as a kind of field bus applied to the special occasion of train control, is widely used in the field of rail transit. The TCN train communication network coordinates the work of various vehicles and equipment to ensure safe, stable and comfortable train operation. The generation and development of TCN is of great significance to the railway and urban rail transit communication network. During the operation of the train, there will be a lot of information about the working status

* Corresponding author: cuijinxue@tongji.edu.cn 
of the on-board equipment, fault records and operation control. The TCN train communication network makes this information safe and fast. And efficient transmission and exchange between various on-board equipment. The TCN train communication network consists of two layers of buses, namely Wire Train Vehicle (WTB) and Multifunction Vehicle Bus (MVB). MVB is applied to the communication network of carriages or fixed formations, and is currently widely used in urban rail transit [3].

According to the provisions of the TCN standard, the MVB bus can use three different physical media and drive methods, namely, ESD (Electrical Short Distance Medium) without electrical isolation, and EMD (Electrical Middle-Distance Medium) with isolation, long-distance optical medium OGF (Optical Glass Fibre medium), the data transmission speed is $1.5 \mathrm{Mbps}$. At present, EMD is widely used in the field of urban rail transit.

The data transmission in the MVB network adopts the form of unicast or broadcast, and the encoding form adopts Manchester encoding. The negative transition in the middle of the bit means " 0 ", and the negative transition in the middle of the bit means "1". The message on the MVB bus consists of a master frame and a slave frame [4]. The structure of the message is shown in the figure below. The master frame is sent by the bus manager (BA) according to the cycle schedule. The master frame contains 9 bits of the start identifier identification code, 4 bits of F-code code indicating the type and size of the slave frame, 12 bits of the slave device address, and $8 \mathrm{CRC}$ check. Bit, the end identifier is 1 bit. The slave frame contains 9 bits of the start identifier identification code, 16-256 bits of data field and an additional 8-bit check sequence for every 64 bits [5].

\section{MVB conformance test}

Conformance testing refers to the degree of compliance between the implementation of the test protocol and the protocol specification standards. It is a process that can determine whether a communication device complies with the technical requirements of the standard and demonstrates the performance required by the standard. The standards of communication protocols are usually very complicated, and it is difficult to conduct thorough tests in all aspects. Therefore, conformance testing does not provide thorough tests on every aspect of the standard. Since the conformance test is completed in a controlled environment, in fact, a device that has passed the conformance test cannot theoretically guarantee interoperability with any other device that has passed the conformance test. This is mainly because many other factors that affect the operation of the protocol, such as network load and timeout regulations, will affect the performance of the IUT and have an impact on interoperability. But in fact, the prospects for successful interconnection of devices that have passed the conformance test are indeed improved. The interoperability between devices that have passed the conformance test is relatively easy to achieve. Therefore, the conformance test is necessary for communication devices to achieve interoperability. Condition [6].

But in fact, the prospects for successful interconnection of devices that have passed the conformance test are indeed improved. The interoperability between devices that have passed the conformance test is relatively easy to achieve. Therefore, the conformance test is necessary for communication devices to achieve interoperability [7].

According to IEC61375-3-2, the MVB physical layer conformance test includes resistance test, inductance test, measurement of insertion loss, measurement of signal waveform during transmission (heavy test circuit, light test circuit, idling test circuit and short test circuit), receiver behaviour test threshold $200 \mathrm{mV}$ and $500 \mathrm{mV}$.

The resistance test is a pure resistance test for the termination resistance of the A line and B line of the MVB, where the IUT is powered off and the impedance should be $120 \Omega$ range $\pm 10 \%$. Inductance testing is to measure the inductance of the device under test 
when the IUT is powered off. The maximum permissible phase displacement is $0,087 \mathrm{rad}$ in a frequency range between 0,5 and 2,0 BR. Measurement of insertion loss is to measure the insertion loss of the controller at different frequencies $(0,5 \mathrm{BR} \sim 2,0 \mathrm{BR})$ when the IUT is powered off or in normal operation. The maximum allowable loss is required to be less than $0.15 \mathrm{~dB}$. The measurement of signal waveform during transmission is to check whether the output waveform under four circuit situations meets the standard IEC61375-3-2 requirements. The four circuit situations are shown in Figure 1 ( $\mathrm{Zt}$ is $120 \Omega$ ).

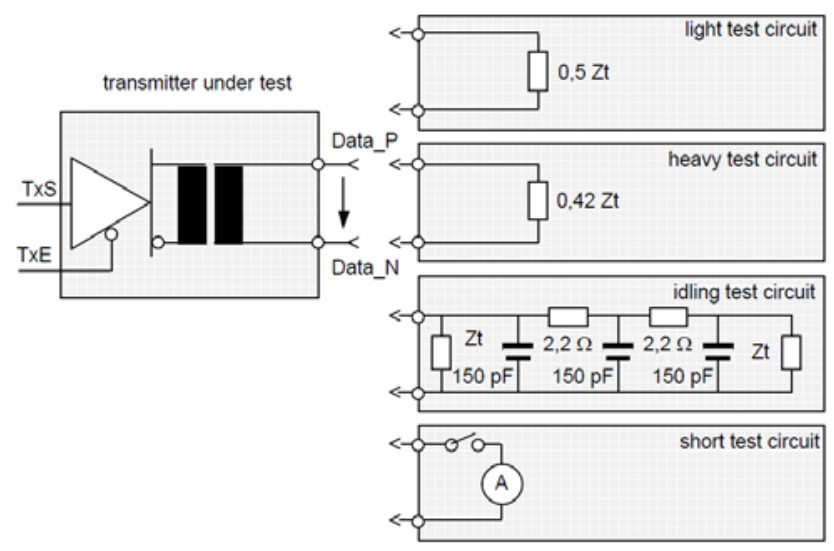

Fig. 1. EMD transmitter test circuits.

\section{MVB conformance test system}

The MVB conformance test system is composed of two layers, the first layer is to build the MVB network required for testing, and the second layer is the test instrument. The testing equipment adopts micro resistance tester, LCR tester, vector network analyser and oscilloscope. The structure diagram is shown in Figure 2.

First set up the MVB network required for testing. After completing the setup, connect to the test instrument to collect the test information, and then transmit it to the upper computer. The upper computer processes and analyses the data, compares it with the data required by the standard, and judges the data does it reach the requirement.

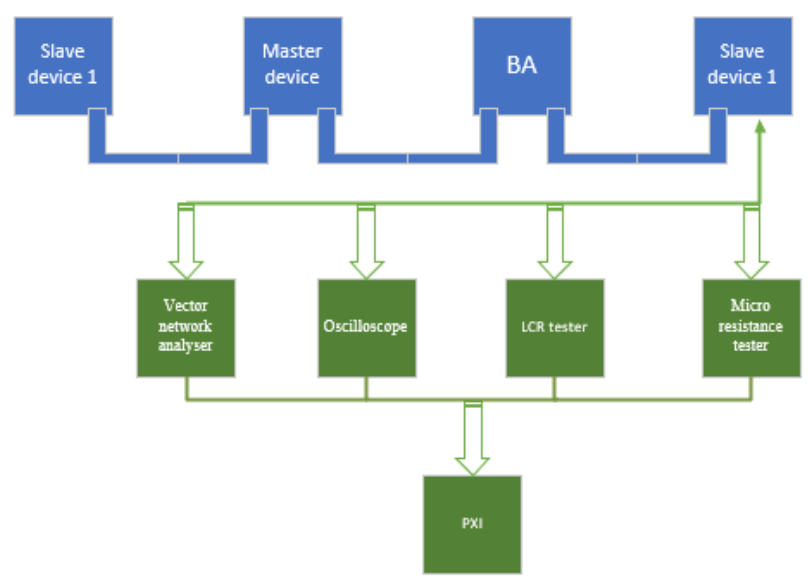

Fig. 2. MVB conformance test system. 


\section{MVB network for testing}

The MVB network required for the test consists of three devices, a master device with BA function and two slave devices, as shown in Figure 3 and Figure 4.

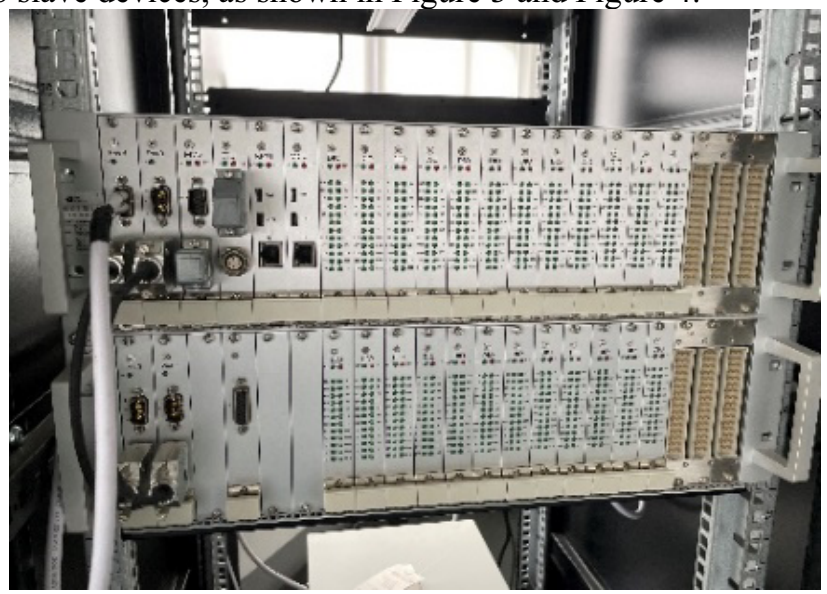

Fig. 3. Slave device 2 .

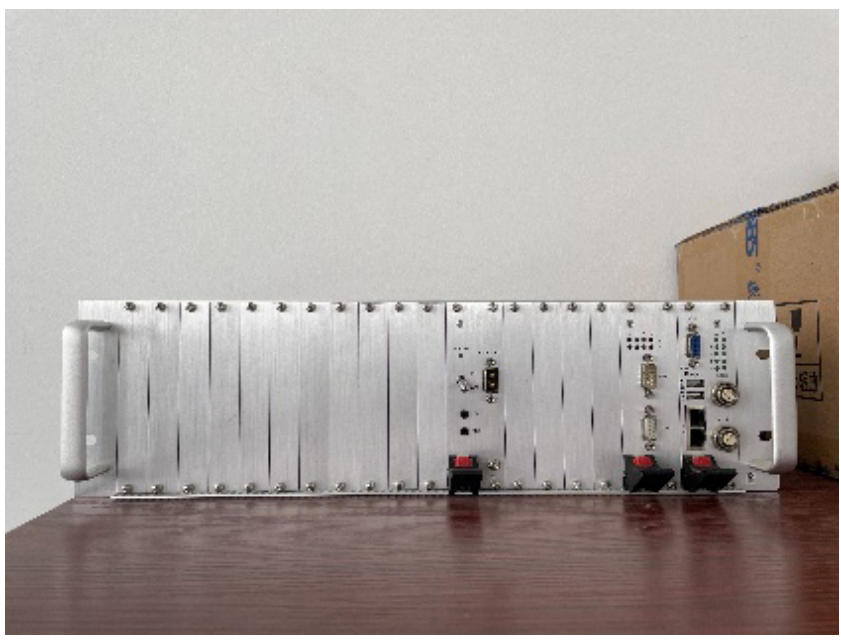

Fig. 4. Master device.

According to the test requirements and follow-up development needs, the ports of the three devices are configured as shown in Figure 5. The interface of related information displayed in slave device 1 is shown in Figure 6.

According to the period of sending and receiving data, a periodic schedule is established. The schedule is shown in Figure 7. The scheduling of period information is realized by referring to the single-processor real-time task scheduling algorithm. Use the least common multiple/greatest common divisor ( $\mathrm{LCM} / \mathrm{HCF}$ ) method to determine the size of the MVB real-time schedule [8]. The synchronous RM scheduling algorithm is used to allocate the corresponding priority of each MVB period information to perform non-pre-emptive realtime scheduling, generate the corresponding scheduling table, and realize the communication between the devices. Figure 8 shows the interface for establishing communication between devices. 
Slave device 1 is a control workstation with display function, which is responsible for displaying the network structure and realizing network port configuration functions in the MVB network. The interface design of this device is shown in Figure 9, which shows the basic information of the MVB network required for testing. MVB physical layer conformance test system will conduct test research based on this MVB network.

\begin{tabular}{|c|c|c|c|c|c|c|}
\hline \multirow{2}{*}{ Address description } & \multirow{2}{*}{ Device address } & \multicolumn{2}{|c|}{ Portaddress } & \multirow{2}{*}{ F-code } & \multirow{2}{*}{ Portsize/byte } & \multirow{2}{*}{ Characteristic period/ms } \\
\hline & & Hexadecimal & Decimal & & & \\
\hline \multirow{4}{*}{ Portaddress from slave device 1 to $\mathbb{m}$ asterdevice } & \multirow{4}{*}{$0 \times 21$} & 210 & 528 & 4 & 32 & 256 \\
\hline & & 211 & 529 & 4 & 32 & 256 \\
\hline & & 212 & 530 & 4 & 32 & 256 \\
\hline & & 213 & 531 & 4 & 32 & 256 \\
\hline \multirow{3}{*}{ Port address from slave device 2 to m asterdevice } & \multirow{3}{*}{$0 \times 3 \mathrm{H}$} & $3 \mathrm{AO}$ & 928 & 4 & 32 & 512 \\
\hline & & $3 \mathrm{H}$ & 928 & 4 & 32 & 512 \\
\hline & & $3 \mathrm{H} 2$ & 928 & 4 & 32 & 512 \\
\hline \multirow{6}{*}{ 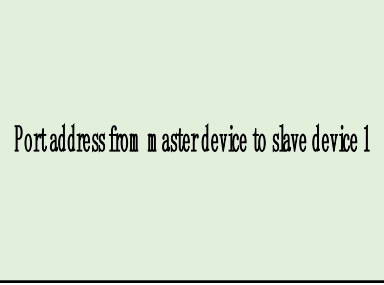 } & \multirow{6}{*}{ 0xil } & 201 & 513 & 4 & 32 & 256 \\
\hline & & 202 & 514 & 4 & 32 & 1024 \\
\hline & & 203 & 515 & 4 & 32 & 256 \\
\hline & & 204 & 516 & 4 & 32 & 256 \\
\hline & & 205 & 517 & 3 & 16 & 64 \\
\hline & & 206 & 518 & 4 & 32 & 64 \\
\hline II asterderice to allW VB devices & Ox11 & Ff & 255 & 3 & 16 & 64 \\
\hline \multirow{2}{*}{ Port address from a asterderice to save device 2} & \multirow{2}{*}{ oxl1 } & 301 & 769 & 3 & 16 & 64 \\
\hline & & 302 & 770 & 4 & 32 & 64 \\
\hline
\end{tabular}

Fig. 5. Network structure.

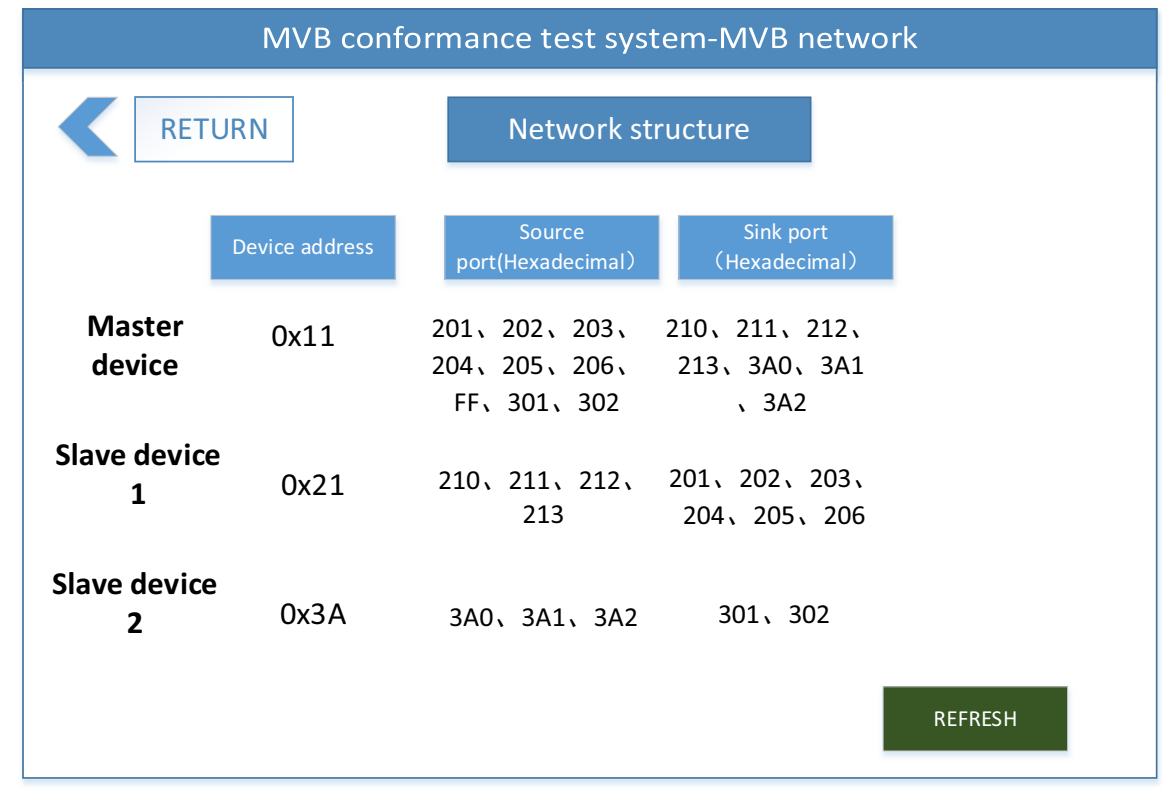

Fig. 6. The interface of related information displayed in slave device 2. 


\begin{tabular}{|c|c|c|c|c|}
\hline M icrocycle & $\begin{array}{c}\text { Port address for } \\
\text { sending data } \\
\text { (hexadecim al) }\end{array}$ & Address descrip tion & F-code & Characteristic period/m s \\
\hline \multirow{2}{*}{$1 \mathrm{~m} \mathrm{~s}$} & $\mathrm{FF}$ & M aster device to allM VB devices & 3 & 64 \\
\hline & 302 & M aster device to slave device 2 & 4 & 64 \\
\hline \multirow{2}{*}{$1 \mathrm{~m} \mathrm{~s}$} & 301 & M aster device to slave device 2 & 3 & 64 \\
\hline & 206 & M aster device to slave device 1 & 4 & 64 \\
\hline \multirow{2}{*}{$1 \mathrm{~m} \mathrm{~s}$} & 205 & M aster device to slave device 1 & 3 & 64 \\
\hline & 201 & M aster device to slave device 1 & 4 & 256 \\
\hline \multirow{2}{*}{$1 \mathrm{~m} \mathrm{~s}$} & 203 & M aster device to slave device 1 & 4 & 256 \\
\hline & 204 & M aster device to slave device 1 & 4 & 256 \\
\hline \multirow{2}{*}{$1 \mathrm{~m} \mathrm{~s}$} & 210 & Slave device 1 to $\mathrm{m}$ aster device & 4 & 256 \\
\hline & 211 & Slave device 1 to $\mathrm{m}$ aster device & 4 & 256 \\
\hline \multirow{2}{*}{$1 \mathrm{~m} \mathrm{~s}$} & 212 & Slave device 1 to $\mathrm{m}$ aster device & 4 & 256 \\
\hline & 213 & Slave device 1 to $\mathrm{m}$ aster device & 4 & 256 \\
\hline \multirow{2}{*}{$1 \mathrm{~m} \mathrm{~s}$} & $3 \mathrm{~A} 0$ & Slave device 2 to $\mathrm{m}$ aster device & 4 & 512 \\
\hline & $3 \mathrm{~A} 1$ & Slave device 2 to $\mathrm{m}$ aster device & 4 & 512 \\
\hline \multirow{2}{*}{$1 \mathrm{~m} \mathrm{~s}$} & $3 \mathrm{~A} 2$ & Slave device 2 to $\mathrm{m}$ aster device & 4 & 512 \\
\hline & 202 & M aster device to slave device 1 & 4 & 1024 \\
\hline
\end{tabular}

Fig. 7. Periodic schedule.

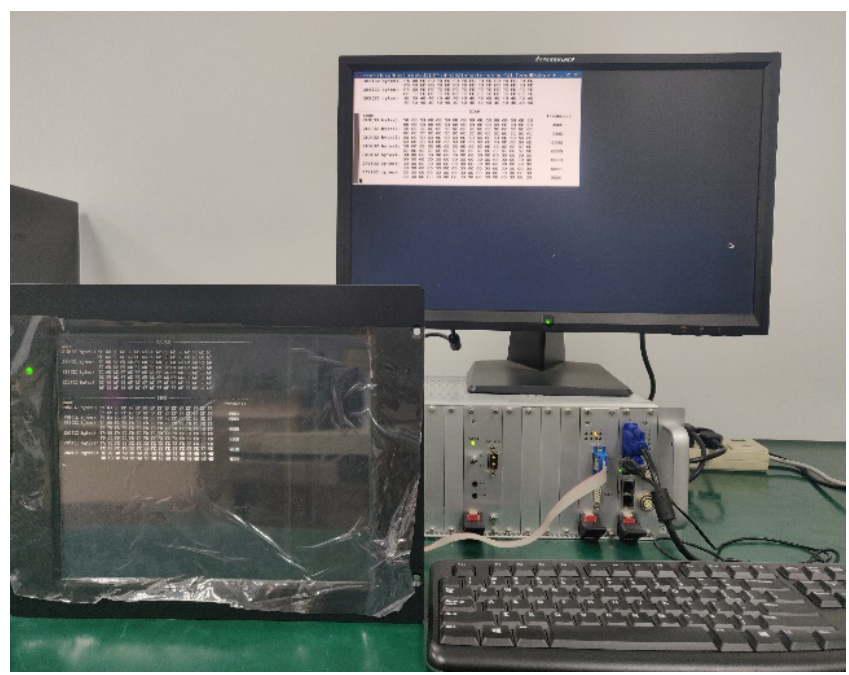

Fig. 8. The interface for establishing communication between devices. 


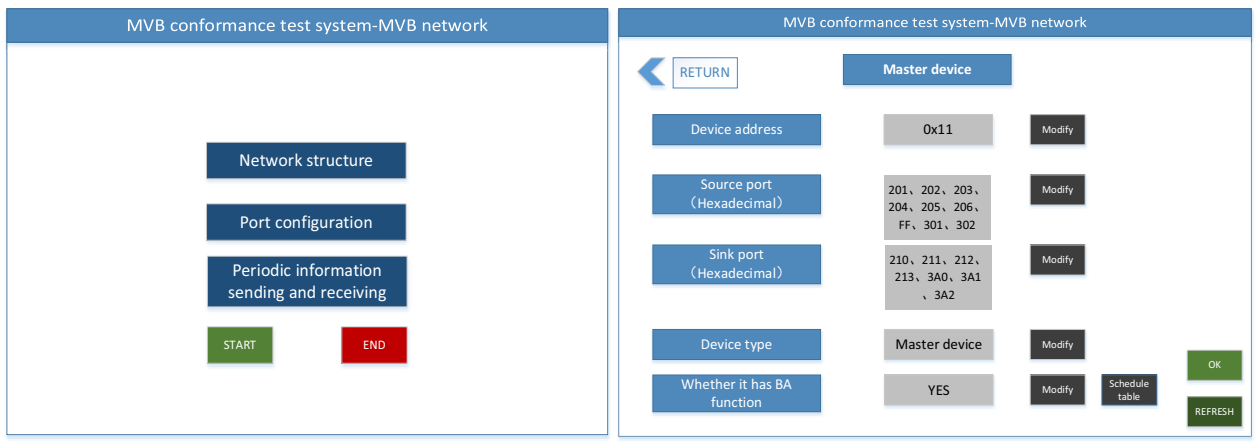

Fig. 9. The interface design of slave device 1 .

\section{Conclusion}

As a train network widely used in urban rail transit, MVB plays a very important role in the safety, comfort and intelligence of urban rail transit. In order to ensure good interoperability and compatibility between train equipment produced by different countries and different manufacturers, conformance testing must be carried out. The formation of the MVB physical layer conformance test system is of great significance for subsequent conformance tests of RTP real-time protocols such as the MVB link layer, network layer, transport layer and application layer.

This work was supported by the National Key R\&D Program of China(2019YFB1600702) and the National Key R\&D Program of China (2020YFB1600705).

\section{References}

1. Li C X and Xie B M 2006 Self development of train communication network technology Electric Drive for Locomotives 02 10-13.

2. GB/T 28029.10 2020 Electronic railway equipment - Train communication network (TCN) - Part 3-2: Multifunction Vehicle Bus (MVB) conformance testing.

3. LIU J, TAN N, JIAO F, et al. Performance Analysis of Multifunction Vehicle Bus System $[\mathrm{J}][\mathrm{J}]$. Journal of the china railway society, 2006, 6: 017.

4. Zhao W H and Zhu G C 2009 Research and Development of MVB Communication Network Card RAILWAY LOCOMOTIVE \&CAR 04 30-33

5. ZHU J, LI F, WANG L. Research on Network Dynamic Performance of Multifunction Vehicle Bus[J]. Electric Drive for Locomotives, 2013: 01.

6. ZHU Q, XIE W, TAN X. Research on MVB Protocol Conformance Testing [J][J]. Journal of the China Railway Society, 2007, 4: 115-120.

7. XU L, ZHANG J, LIU B. Design of Protocol Conformance Testing System Based on MVB[J]. Control Engineering of China, 2014: 01.

8. Zhu Q Y and Xie W D 2007 Real-time scheduling of periodic message for MVB Computer application 12 3108-3111+3115 